\title{
Glucose Transporter 1 Expression Is Regulated by Aged Garlic Extract during Cerebral Ischemia
}

\author{
Mónica Espinoza-Rojo ${ }^{1}$, Carlos D. Gómez ${ }^{1,2,3}$, Juan Parra Abarca ${ }^{1}$, Alma Ortiz-Plata ${ }^{4}$, \\ Martha-Eugenia Ruiz-Tachiquín ${ }^{5}$, JoséPedraza-Chaverri ${ }^{6}$, Pavel Montes de Oca Balderas ${ }^{7}$, Penélope Aguilera ${ }^{2, *}$ \\ ${ }^{1}$ Laboratorio de Biología Molecular y Genómica, Universidad Autónoma de Guerrero, Chilpancingo, Guerrero, MÉXICO \\ ${ }^{2}$ Laboratorio de Patología Vascular Cerebral, Departamento de Neuroquímica. Instituto Nacional de Neurología y Neurocirugía \\ "Manuel Velasco Suárez", México, MÉXICO \\ ${ }^{3}$ Departamento de Biología Celular y Fisiología, Instituto de Investigaciones Biomédicas, Universidad Nacional Autónoma de México, \\ México, MÉXICO \\ ${ }^{4}$ Laboratorio de Neuropatología Experimental, Departamento de Neuroquímica. Instituto Nacional de Neurología y Neurocirugía \\ "Manuel Velasco Suárez", México, MÉXICO \\ ${ }^{5}$ Unidad de Investigación Médica en Genética Humana, Hospital de Pediatría $2^{\circ}$ Piso, Centro Médico Nacional Siglo XXI, México, \\ MÉXICO \\ ${ }^{6}$ Departamento de Biología, Facultad de Química, Universidad Nacional Autónoma de México, México, MÉXICO \\ ${ }^{7}$ Unidad de Neurobiología Dinámica, Departamento de Neuroquímica. Instituto Nacional de Neurología y Neurocirugía "Manuel \\ Velasco Suárez", México, MÉXICO \\ *Corresponding author: penelope.aguilera@gmail.com
}

Received August 22, 2014; Revised October 28, 2014; Accepted November 07, 2014

\begin{abstract}
Aged garlic extract is obtained after prolonged hydroethanolic extraction of garlic cloves and is recognized for its high antioxidant content. Although evidence indicates that aged garlic extract delays brain ischemia-induced damage, the molecular mechanisms of this effect are unclear. Moderate concentration of reactive oxygen species induces glucose transporter 1 (GLUT1) expression that in astrocytessupports brainenergy metabolism. This has suggested that reduction of excessive reactive oxygen species formation during severe ischemia maycontribute to neuroprotection. Thus, in view of the high antioxidant properties of aged garlic extract, we investigated the effect of ischemia on GLUT1 expression and its regulation after aged garlic extract treatment. Rats were subjected to middle cerebral artery occlusion during 2 hto produce ischemia and then were sacrificed after different times of reperfusion. In fronto-parietal cortex GLUT1 mRNA expression peaked after 1and 24h of reperfusion. Nevertheless, no significant change on total protein level was observed by Western blotting, buta severe reduction on GLUT1 positive astrocytes was detected by immunofluorescence after $2 \mathrm{~h}$ of reperfusion. Remarkably, aged garlic extract ( $360 \mathrm{mg} / \mathrm{kg}$;i.p.) administered after is chemia increased GLUT1 mRNA expressionin cortex after $2 \mathrm{~h}$ of reperfusion and partially prevented the middle cerebral artery occlusion-induced reduction of GLUT1 in astrocytes. These results suggest that GLUT1 over expression in astrocytes may underlie some of the beneficial effects on neuronal survival observed with aged garlic extract treatment.
\end{abstract}

Keywords: aged garlic extract, glucose transporter, antioxidants, cerebral ischemia, astrocytes

Cite This Article: Mónica Espinoza-Rojo, Carlos D. Gómez, Juan Parra Abarca, Alma Ortiz-Plata, MarthaEugenia Ruiz-Tachiquín, JoséPedraza-Chaverri, Pavel Montes de Oca Balderas, and Penélope Aguilera, "Glucose Transporter 1 Expression Is Regulated by Aged Garlic Extract during Cerebral Ischemia." Journal of Food and Nutrition Research, vol. 2, no. 12 (2014): 899-905. doi: 10.12691/jfnr-2-12-7.

\section{Introduction}

Excessive production of reactive oxygen species (ROS) by mitochondria contributes significantly to the early stages of cell death during cerebral ischemia. When blood flow is interrupted, decreased ATP level alters cell membrane potential leading to an uncontrolled release of the excitatory neurotransmitter glutamate. This results in an increased influx of calcium, which causes mitochondrial calcium overload followed by production of harmful levels of ROS [1]. Antioxidant therapies have been considered a potential approach to ameliorate ischemic-induced injury due to their primary ability to reduce oxidative stress. Additionally, it has been demonstrated that low levels of ROS trigger intrinsic protective signaling [2]. Thus, besides reducing ROS harmful levels, antioxidants may allow the activation of signaling pathways that induce post-translational modification of proteins and gene regulation that is critical for cellular adaptation to oxygen and glucose deprivation (OGD).

Glucose, the main energy substrate in brain, is taken up into cells by facilitated diffusion, mediated by members of the glucose transporter (GLUT) family [3]. The GLUT1 isoform, abundantly expressed in astrocytes, is essential 
for the adequate supply of glucose to neurons since these cells are the interphase between the vascular system and neurons[4]. In pathological conditions, astrocytes modify their gene expression helping to support neighboring neurons. In particular, GLUT1 over expression enhances glucose uptake by astrocytes increasing lactate production, which is delivered to neurons contributing to their survival $[5,6,7]$. Since GLUT1 gene responds to OGD [3,8] we hypothesized that antioxidants could regulate GLUT1 expression in the brain under ischemic conditions helping to neuronal survival.

Garlic has been used in traditional medicine due to its diverse biological activities that include a main antioxidant effect. Aged garlic extract (AGE) is an odorless product obtained after aged of fresh garlic on ethanol for at least two years[9]. Despite that AGE's antioxidant activity has been associated to its protective effect on cerebral ischemia $[10,11]$, the molecular mechanisms underlying AGE's protection have not been fully elucidated Therefore, in this work, we established the temporal effect of ischemia and restoration of blood flow (reperfusion) in GLUT1 mRNA and protein expression in fronto-parietal cortex of Wistar rats. In addition, we evaluated the effect of AGE treatment on GLUT1 expression in glial fibrillary acidic protein (GFAP) positive astrocytes in cerebral cortex.

\section{Materials and Methods}

\subsection{Reagents}

Aged garlic extract (AGE) Kyolic $\AA$ was obtained from Wakunaga of America Co., Ltd. (Mission Viejo, CA, USA). TRIzol Reagent was from Invitrogen Life Technologies; M-MLV reverse transcriptase, random hexamer primers, GLUT1 (Rn00684729_g1) and 18S ribosomal RNA (18S rRNA) (4308329) were from Applied Biosystems. Mixes of phosphatase and protease inhibitors were from SigmaAldrich. Mouse anti GLUT1 (07-1401) antibody used for western blotting (WB) was from Millipore, rabbit anti GLUT1 (sc-7903) antibody used for immunofluorescence was from Santa Cruz Biotechnology and mouse anti GFAP (GA-5) antibody was from BioGenex. All secondary antibodies were from Jackson Inmuno Research. All other reagents were obtained from known commercial sources.

\subsection{Treatment}

A single dose of AGE $(360 \mathrm{mg} / \mathrm{kg}$; i.p.) was injected after ischemia, and $2 \mathrm{~h}$ later its effect was evaluated $[10,12]$. Control groups pre-administered with AGE were sacrificed by decapitation $2 \mathrm{~h}$ after AGE injection.

\subsection{Experimental Animals}

Adult male Wistar rats weighing 280-320 g were included in the present study $(n=4-6)$. Experimental procedures were carried out in accordance with the ethical guidelines established by the NIH Guidelines for the Care and Use of Laboratory Animals and by the Instituto Nacional de Neurología y Neurocirugía "Manuel Velasco Suárez" [approved project No. 20/11 (624)].

\subsection{MCAO Model and Behavioral Testing Evaluation}

Animal were subjected to middle cerebral artery occlusion (MCAO) during $2 \mathrm{~h}$, as described by Longa et al. $[12,13]$, and were sacrificed by decapitation after different times of reperfusion $(0,1,2,3,4,6,10,24$, or 48 h). Briefly, animals were anaesthetized with isoflurane (2.5$3.0 \%$ ). The left common carotid artery was exposed at the level of the external and internal carotid artery bifurcation. A 3-0 nylon monofilament was inserted into the external carotid artery and advanced into the internal carotid artery for a length of about $17 \mathrm{~mm}$ in order to block the origin of the MCA. Body temperature was kept at $37 \pm 0.5^{\circ} \mathrm{C}$ during the whole procedure. Two h after the induction of ischemia, the filament was removed to allow reperfusion. A control group was subjected to surgery without MCAO. Animals were then returned to their cages and monitored until they recovered from anesthesia. Neurological deficit was determined 30 min before reperfusion and was scored on a 2-point scale: failure to extend right paw fully $=1$; circling to right (more than 5 turns over a period of $30 \mathrm{sec}$ ) $=1$. Animals that presented neurological deficits $<2$ were exclude from the study $[10,12]$.

\subsection{Quantitative Reverse Transcription PCR (qPCR)}

For isolating the RNA, the whole fronto-parietal cortex was dissected. Total RNA extraction was performed using TRIzol reagent according to the manufacturer's instructions. The cDNA was synthesized from $5 \mu \mathrm{g}$ total RNA by using $200 \mathrm{U}$ of M-MLV reverse transcriptase and $2.5 \mu \mathrm{M}$ of random hexamer primers. Subsequently, the cDNA was used as template to evaluate relative mRNA expression. qPCR was performed in a 7500 Real-time PCR System (Applied Biosystems) using a specific assay for GLUT1 and 18S rRNA was used as a control to normalize the relative mRNA amount of the target gene. Reactions were done in triplicate and consisted in a denaturation cycle at $95^{\circ} \mathrm{C}$ for $10 \mathrm{~min}$, followed by 40 cycles of denaturation at $92^{\circ} \mathrm{C}$ for $15 \mathrm{sec}$ and of annealing/extension at $60^{\circ} \mathrm{C}$ for $1 \mathrm{~min}$. Values of threshold cycle $(\mathrm{Ct})$ were determined by automated threshold analysis using SDS Software 1.3.1. This software uses the comparative $\Delta \Delta \mathrm{Ct}$ method of relative quantification to determine relative gene expression levels.

\subsection{Western Blotting (WB)}

Whole fronto-parietal cortex was dissected and homogenized in lysis buffer (Tris- $\mathrm{HCl} 50 \mathrm{mMpH}$ 7.5, $\mathrm{NaCl} 150 \mathrm{mM}$, EDTA $1 \mathrm{mM}$ ) containing a mix of phosphatase and protease inhibitors. Lysates were assessed for protein concentration using Lowry protein assay. Protein was boiled in Laemmli sample buffer. Twenty $\mu \mathrm{g}$ of protein were resolved in $10 \%$ SDSpolyacrylamide gels. Proteins were transferred to nitrocellulose membranes. Membranes were blocked with PBS containing 5\% dry skimmed milk and incubated over night at $4^{\circ} \mathrm{C}$ with anti GLUT1 antibody $(1: 1000)$ and for 1 $h$ with anti-rabbitIgG-HRP antibody $(1.20,000)$ and revealed by chemiluminescence. 


\subsection{Immunofluorescence}

Animals were anesthetized with pentobarbital (63 $\mathrm{mg} / \mathrm{kg}$ ) intramuscularly and perfused via the ascending aorta with $250 \mathrm{~mL}$ of cold $0.1 \mathrm{M}$ phosphate buffered saline (PBS), $\mathrm{pH} 7.4$, followed by $250 \mathrm{~mL}$ of $4 \%$ paraformaldehyde in PBS. The brains were removed, postfixed for $24 \mathrm{~h}$ with $4 \%$ paraformaldehyde and cryoprotected in $30 \%$ sucrose in PBS for $48 \mathrm{~h}$. Brain tissue was cut into $10 \mu \mathrm{m}$ thick sections using a cryostat (Microm HM 520, Thermo Scientific). Brain slices were mounted on slides coated with gelatin and dried at $56^{\circ} \mathrm{C}$ during $30 \mathrm{~min}$. Then, brain sections were incubated in PBS containing 2\% BSA and $0.5 \%$ Tween 20 for $30 \mathrm{~min}$. The sections were incubated with rabbit anti GLUT1 (1:50) and mouse anti GFAP (1:250) at $4^{\circ} \mathrm{C}$ overnight, followed by incubation with anti-rabbit IgG-DyLight 594 (1:300) and anti-mouse IgG-DyLight 488 (1:300) at room temperature for $90 \mathrm{~min}$ in the dark. PBS containing $10 \%$ BSA and $0.3 \%$ Triton-X100 was used to dilute the antibodies and PBS was used to wash the sections between each step. Finally, sections were labeled with 4',6-diamidino-2-phenylindole (DAPI, 1 $\mu \mathrm{g} / \mathrm{mL}$ ) for $15 \mathrm{~min}$ at room temperature in the dark. A serial of 6-7 fields $(\times 40)$ were acquired per slide located at the cortex (contralateral and ipsilateral to lesion) using an epifluorescence microscope (Olympus model 1X81) coupled to a digital camera Hamamatsu Orca R2. The percentage of immunopositive cells was determined using the ImageJ program (imagej.nih.gov) establishing a common threshold minimal intensity value to claim positive staining. The number of GLUT1-positive astrocytes was normalized by the number of DAPI stained nuclei and expressed as percentage.

\subsection{Statistical analysis}

Data are presented as mean and standard deviation (mean \pm SD). Analysis of variance (ANOVA) and post hoc Tukey analysis were used for statistical evaluations. Data analysis was performed using SPSS 13.0 (SPSS Inc., Chicago, IL, USA) statistical package software.

\section{A}

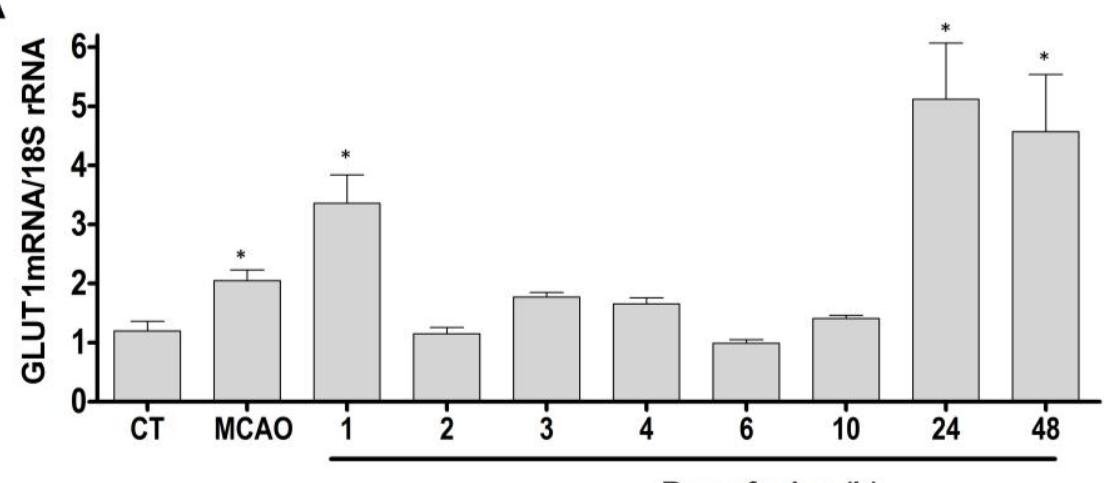

B

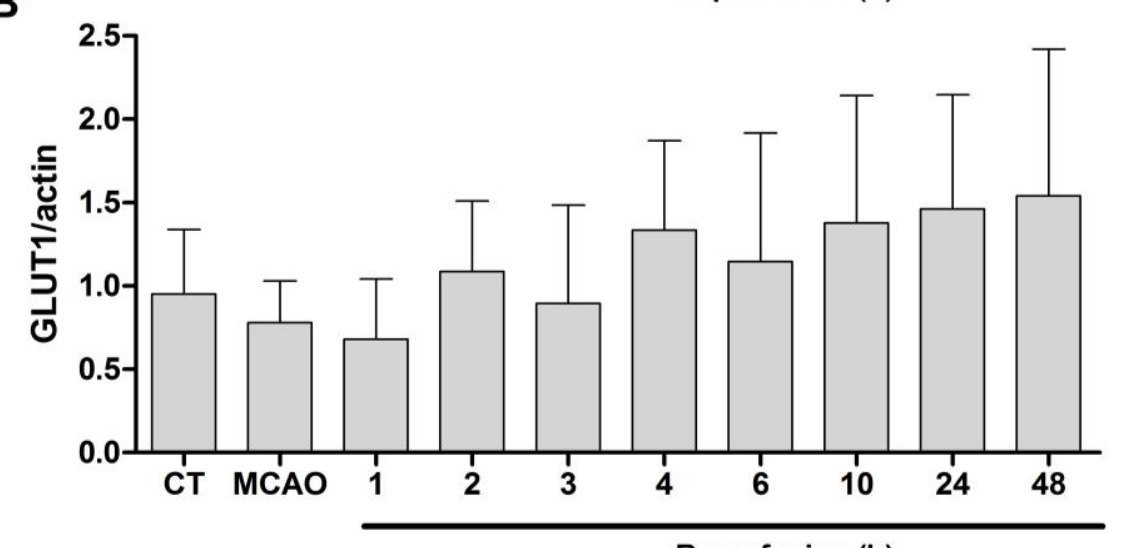

C

Reperfusion (h)

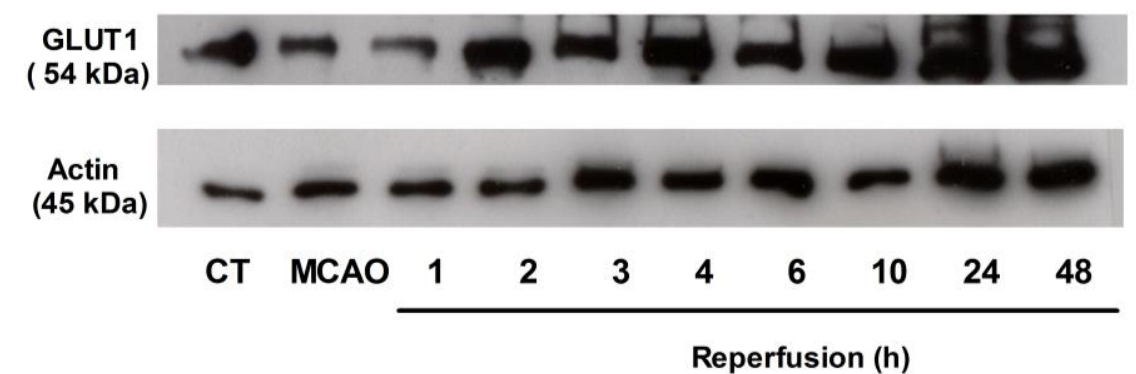

Figure 1. Effect of middle cerebral artery occlusion (MCAO)/reperfusion on glucose transporter 1 (GLUT1) mRNA and protein expression. Rats were subjected to MCAO for $2 \mathrm{~h}$, and sacrificed at the specified times of reperfusion. mRNA level was determined using qPCR (A) and protein levels by WB $(\mathrm{B}, \mathrm{C})$. Mean $\pm \mathrm{SD}(\mathrm{n}=4-6)$. One way-ANOVA, Tukey $* P<0.05$ vs control $(\mathrm{CT})$ 


\section{Results and Discussion}

\subsection{Ischemia/reperfusion Increases GLUT1 mRNA Expression}

In order to evaluated the effect of ischemia and reperfusion, animals were subjected to transient MCAO during $2 \mathrm{~h}$, and were sacrificed after different times of reperfusion $(0,1,2,3,4,6,10,24$, or $48 \mathrm{~h})$. As shown in Figure $1 \mathrm{~A}, \mathrm{MCAO}$ without reperfusion increased $71 \%$ the baseline expression of GLUT1 mRNA. After $1 \mathrm{~h}$ of reperfusion GLUT1 mRNA expression showed a $180 \%$ increase, over baseline. Twoh after reperfusion, GLUT1 mRNA returned to control levels and no further changes were observed after $3,4,6$ or $10 \mathrm{~h}$ of reperfusion. Interestingly, we found GLUT1 mRNA expression peaked again after $24(327 \%)$ and $48(281 \%)$ h of reperfusion. Nevertheless, we found that the increased GLUT1 mRNA levels induced by $\mathrm{MCAO} /$ reperfusion did not result in augmented GLUT1 protein levels evaluated by WB (Figure 1B).

It is possible that the immediate GLUT1 mRNA increment observed after $1 \mathrm{~h}$ of reperfusion could involve the activation of proteins that induce transcription, such as the hypoxia inducible factor 1 (HIF-1). This is because the lack of oxygen strongly up-regulates GLUT1 mRNA expression in HIF-1 dependent manner [8]. Accordingly, it has been shown that OGD increases expression of a reporter gene under the control of a cis-acting regulatory element located in the GLUT1 3'-untranslated region [14] and thatGLUT1 mRNA increment induced by hypoxia is blocked by siRNA against HIF-1 $\alpha[15,16]$.

The second peakon GLUT1 mRNA expression,detected after 24 and $48 \mathrm{~h}$ of reperfusion,is consistent with previous Northern blot and in situ hybridization analysis that found increased GLUT1 mRNA transcription after MCAO and $>12 \mathrm{~h}$ of reperfusion [17]. These data suggest that GLUT1 mRNA expression is also up-regulated by MCAO after long periods of reperfusion through different mechanisms that could or could not implicate HIF activation $[15,18]$.

On the other hand, we found that GLUT1 protein was not significantly altered after MCAO/reperfusion at all times tested (Figure 1B and Figure 1C). Thiskind of effect may occur during cerebral ischemia, probably associated to glucose utilization in penumbra (region where blood flow is reduced $\sim 40 \%)$, that is elevated $(\sim 50-70 \%)$ at early times of reperfusion, but that gradually fall reaching similar levels than those observed in the core of the lesion ( 25 \%) [19,20]. Therefore, it is possible that despite GLUT1 mRNA is increased initially, brain cells are unable to translate GLUT1 transcripts efficiently at later times. In agreement, in primary cultures of neurons and astroglia, OGD induces GLUT1 mRNA and its protein differently [15,21]. Furthermore, transcription and translation changes are detected only in regions where tissue survives (i.e. penumbra) by increasing glucose transport and glycolysis [22,23]. Therefore, since our analyses were performed in the entire impaired region (i.e. infarct and penumbra) a mix of survival and damaged tissue should exist in our sample. This condition could mask minor or local increments induced by $\mathrm{MCAO} /$ reperfusion on protein expression. In fact, GLUT1 protein level is upregulated after $12 \mathrm{~h}$ of reperfusion in the global ischemia model, in which all brain tissue is subjected to a mild injury [18].

\subsection{AGE Increases GLUT1 mRNA Expression under Control Conditions and after Ischemia/Reperfusion.}

Considering the above resultsand our previous findings in which AGE treatment delayed the appearance of the infarct area for approximately $2 \mathrm{~h}$ after reperfusion[10], we selected this time to evaluate AGE effect on GLUT1 mRNA expression after MCAO. A single dose of AGE was injected at the beginning of reperfusion, and $2 \mathrm{~h}$ later its effect was evaluated. We found that AGE by itself increased GLUT1 transcript level for both groups, control $(156 \%)$ and subjected to MCAO/reperfusion (148\%) (Figure 2). Thus, our experiments showed that AGE administration modifies GLUT1 expression under control and ischemic conditions.

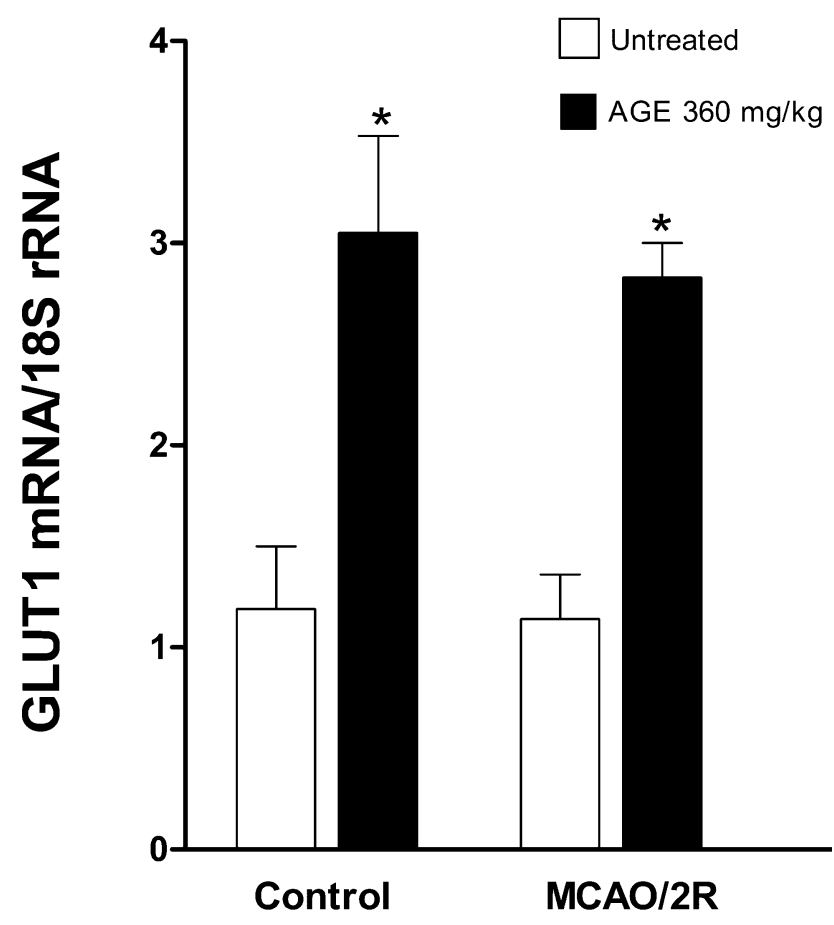

Figure 2. Aged garlic extract (AGE) increases glucose transporter 1 (GLUT1) mRNA expression. Rats were subjected to middle cerebral artery occlusion (MCAO) for $2 \mathrm{~h}$, and sacrificed after $2 \mathrm{~h}$ of reperfusion (R). AGE (360 mg/kg; i.p) was injected at the beginning of reperfusion. mRNA level was determined using qPCR. Mean \pm SD $(n=4-6)$. One way-ANOVA, Tukey $* P<0.05$ vs Control

The effect observed in animals not subjected to $\mathrm{MCAO} /$ reperfusion makes sense with previous works that reported a reduction of blood glucose in response to garlic extracts. For example, in rabbits anethanolycgarlic extract induces a maximum hypoglycemic effect after $4 \mathrm{~h}$ of extract administration [24]. This effect might be mediated in part by GLUT mRNA induction. In this regard, AGE's components might activate a cell-surface receptor associated to a signaling cascade that triggers gene expression. Activation of NADPH oxidase via this putative receptor arouses production of sublethal levels of intracellular ROS that activates cell signaling as a mechanism supplementary 
to agent's antioxidant activity [25]. However, this AGE effect has to be further investigated.

In ischemic groups, AGE might reduce ROS harmful levels and allow activation of mechanisms that induce either GLUT1 mRNA transcription or its stabilization. In accordance, agents that favor a reducing environment (e.g. ROS scavengers) induced HIF- $1 \alpha$ protein stabilization in hypoxic neurons which consequently increase GLUT1 mRNA transcription [26]. Furthermore, AGE alsoincreases $\gamma$-glutamylcystein synthetase mRNA level (our unpublished observations) that supports HIF-1 $\alpha$ protein stabilization [26].

\subsection{AGE Prevents astrocyte's GLUT1 Loss Induced by Ischemia}

In light of these observations, that indicated that AGE has a strong effect on GLUT1 mRNA expression, we evaluated the effect of AGE on GLUT1 protein level in astrocytes, cells critical for neuronal health. This evaluation was performed through double immunofluorescence analysis against GLUT1 and GFAP (Figure 3A). Data showed that MCAO and $2 \mathrm{~h}$ of reperfusion reduced $79.3 \%$ the number of astrocytes (GFAP positive cells) labeled for GLUT1 in the ipsilateral hemisphere of the lesion (Figure 3B and Figure 3C); whereas no changes were observed in the contralateral side to lesion (data not shown). Interestingly, when AGE was administered, the loss of GLUT1 positive astrocytes induced by $\mathrm{MCAO} /$ reperfusion was partially prevented $(65.5 \%)$ (Figure 3C and Figure 3D).Thisis an apparent contradictory result with respect to theunmodified levels of GLUT1 protein observedon fronto-parietal cortexafter MCAO and $2 \mathrm{~h}$ of reperfusion (Figure 1). This differencefound on results could be related to the extent of blood flow reduction in the zone assessed by WBcompared to that evaluated byimmunofluorescence (Figure 3B). It is possible that the zone analyzed by immunofluorescencewas exposed to a severe inhibition of electron transport, decreased ATP levels and free radical production [19]. Consequently, there is reduced protein synthesis in this zone, that it is not appreciated when the wholefronto-parietal cortex is assessedas was done for the WB analysis as discussed above.
A
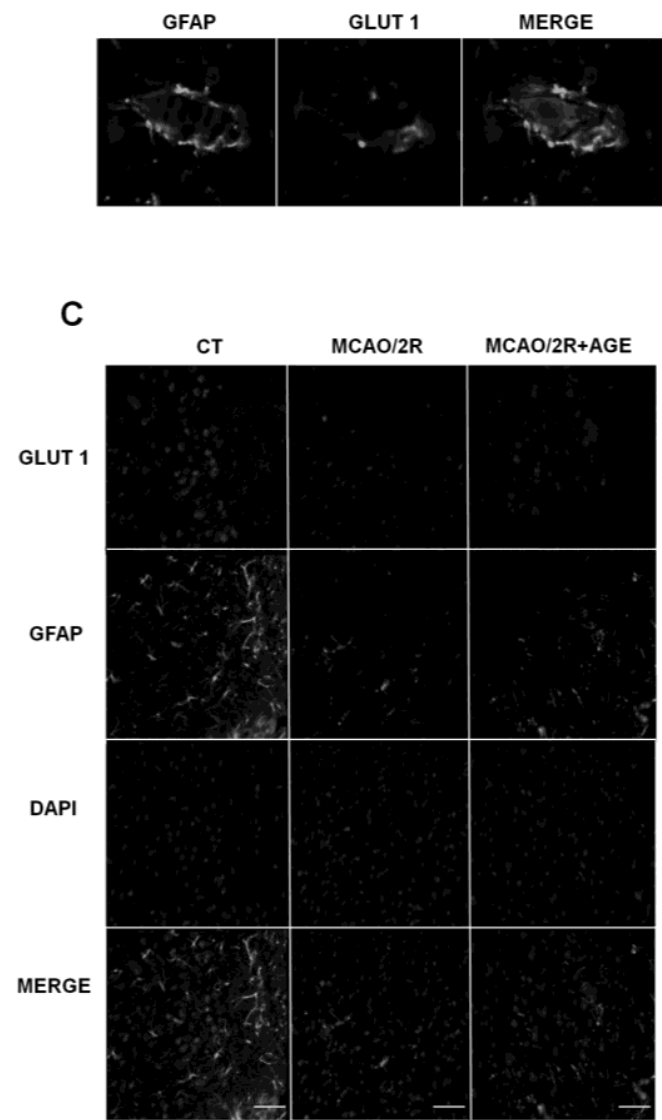

B

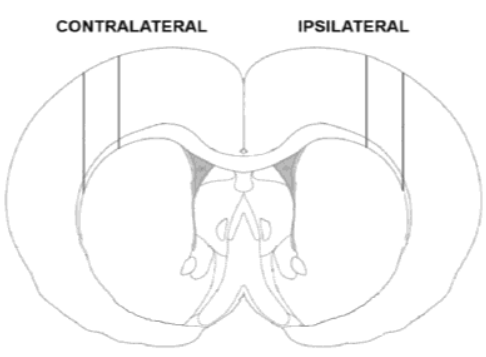

D

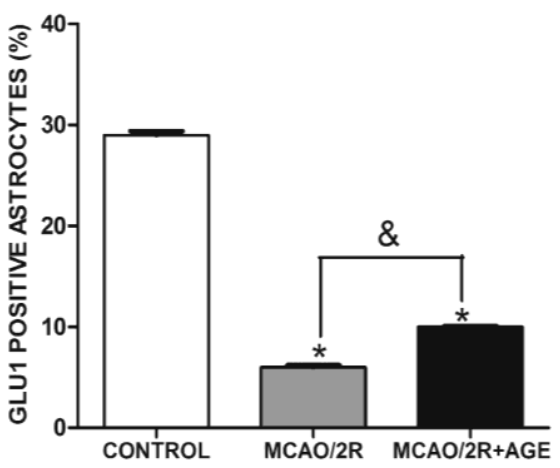

Figure 3. Effect of aged garlic extract (AGE) on glucose transporter 1 (GLUT1) protein expression in glial fibrillary acidic protein (GFAP) positive astrocytes.A. Representative images of double labeled cells with antibodies against GFAP and GLUT1; nuclei stained with DAPI. B. Anatomical position of cortex analyzed (contralateral and ipsilateral to lesion). C. Representative images from ipsilateral series, cells stained with antibody against GLUT1 and GFAP, nuclei stained with DAPI. Reference bar $=10 \mu \mathrm{m}$. D. Quantitative analysis of GLUT1 positive astrocytes (GFAP positive) normalized by the number of DAPI stained nuclei and expressed as percentage. Rats were subjected to middle cerebral artery occlusion (MCAO) for $2 \mathrm{~h}$ and sacrificed after $2 \mathrm{~h}$ of reperfusion. AGE $(360 \mathrm{mg} / \mathrm{kg}$; i.p) was injected at the beginning of reperfusion. One way-ANOVA, Tukey, $* P<0.05 \mathrm{vs}$ control; \& $P<0.05$.

Importantly, AGE effect on GLUT1 protein in astrocytes after MCAO/reperfusion suggests that AGE's mechanism of action could involve an increment on astrocyte glucose uptake. Increased GLUT1 expression in astrocytes might favor trafficking of lactate through the astrocyte-neuron lactate shuttle, allowing neurons to survive more effectively to oxygen deprivation [5]. This is supported by studies with cultured astrocytes showing that 
ischemia induces GLUT3 expression and enhances storage of intracellular glucose during reperfusion [21]. However, it would be helpful to investigate the effect of AGE on GLUT1 expression in cultured astrocytes.

\section{Conclusions}

Ischemia/repefusion increased GLUT1 mRNA expression in brain cortex, effect that was enhanced by AGE. Additionally, AGE treatment prevents GLUT1 protein loss induced by ischemia in astrocytes. It can be speculated that survival pathways responsive to moderate ROS levels (e.g. HIF-1) might contribute to AGE's antioxidant action. Recently, components with a high antioxidant activity were identified in AGE[27]; hence, it would be important to determine those biologically active. Finally, the capability of AGE to up-regulate GLUT1 expression under ischemia/reperfusion might represent a potential advantage in the treatment of stroke in which a high energy demand takes place.

\section{Acknowledgment}

CDGand JPA were financially supported by CONACyT (Scholarship Nos. 12296 and 36883) and by Movilidad Santander Universia 2008 and 2011, respectively. PMOB was supported by CONACyT projects 138425 and 132706 .

\section{Disclosure}

None of the authors has any conflict of interest to disclose.

\section{Abbreviations}

AGE, aged garlic extract; GFAP, glial fibrillary acidic protein; GLUT1, glucose transporter 1; HIF-1, hypoxiainducible factor 1; MCAO, middle cerebral artery occlusion; OGD, oxygen and glucose deprivation; qPCR, quantitative reverse transcription polymerase chain reaction; ROS, reactive oxygen species; WB, Western blotting.

\section{References}

[1] Moskowitz, M.A., Lo, E.H.andIadecola, C., "The science of stroke: mechanisms in search of treatments," Neuron,67 (2). 181-198. Jul 29. 2010.

[2] Thompson, J.W., Narayanan, S.V.andPerez-Pinzon, M.A., "Redox signaling pathways involved in neuronal ischemic preconditioning," Curr Neuropharmacol, 10 (4). 354-369. Dec. 2012.

[3] Espinoza-Rojo, M., Iturralde-Rodriguez, K.I., Chanez-Cardenas, M.E., Ruiz-Tachiquin, M.E. and Aguilera, P., "Glucose transporters regulation on ischemic brain: possible role as therapeutic target," Cent Nerv Syst Agents Med Chem, 10 (4). 317 325. Dec 1. 2010.

[4] Prebil, M., Jensen, J., Zorec, R. and Kreft, M., "Astrocytes and energy metabolism," Arch Physiol Biochem, 117 (2). 64-69. May. 2011.

[5] Cater, H.L., Chandratheva, A., Benham, C.D., Morrison, B., $3^{\text {rd }}$ and Sundstrom, L.E., "Lactate and glucose as energy substrates during, and after, oxygen deprivation in rat hippocampal acute and cultured slices," J Neurochem, 87 (6). 1381-1390. Dec. 2003.

[6] Bliss, T.M., Ip, M., Cheng, E., Minami, M., Pellerin, L., Magistretti, P. and Sapolsky, R.M., "Dual-gene, dual-cell type therapy against an excitotoxic insult by bolstering neuroenergetics," J Neurosci, 24 (27). 6202-6208. Jul 7.2004.

[7] Wohnsland, S., Burgers, H.F., Kuschinsky, W. and Maurer, M.H., "Neurons and neuronal stem cells survive in glucose-free lactate and in high glucose cell culture medium during normoxia and anoxia," Neurochem Res, 35 (10). 1635-1642. Oct. 2010.

[8] Greijer, A.E., van der Groep, P., Kemming, D., Shvarts, A., Semenza, G.L., Meijer, G.A., van de Wiel, M.A., Belien, J.A., van Diest, P.J.andvan der Wall, E., "Up-regulation of gene expression by hypoxia is mediated predominantly by hypoxia-inducible factor 1 (HIF-1)," J Pathol, 206 (3). 291-304. Jul. 2005.

[9] Colin-Gonzalez, A.L., Santana, R.A., Silva-Islas, C.A., ChanezCardenas, M.E., Santamaria, A. and Maldonado, P.D., "The antioxidant mechanisms underlying the aged garlic extract- and Sallylcysteine-induced protection," Oxid Med Cell Longev, 2012 907162. 2012.

[10] Aguilera, P., Chanez-Cardenas, M.E., Ortiz-Plata, A., LeonAparicio, D., Barrera, D., Espinoza-Rojo, M., Villeda-Hernandez, J., Sanchez-Garcia, A. and Maldonado, P.D., "Aged garlic extract delays the appearance of infarct area in a cerebral ischemia model, an effect likely conditioned by the cellular antioxidant systems," Phytomedicine, 17 (3-4). 241-247. Mar. 2010.

[11] Capasso, A., "Antioxidant action and therapeutic efficacy of Allium sativum L," Molecules, 18 (1). 690-700. 2013.

[12] Longa, E.Z., Weinstein, P.R., Carlson, S. and Cummins, R., "Reversible middle cerebral artery occlusion without craniectomy in rats," Stroke, 20 (1). 84-91. Jan. 1989.

[13] Cervantes, M.I., de Oca Balderas, P.M., de Jesus Gutierrez-Banos, J., Orozco-Ibarra, M., Fernandez-Rojas, B., Medina-Campos, O.N., Espinoza-Rojo, M., Ruiz-Tachiquin, M., Ortiz-Plata, A., Salazar, M.I., Rubio-Osornio, M., Castaneda-Saucedo, E., PedrazaChaverri, J., Calzada, F.andAguilera, P., "Comparison of antioxidant activity of hydroethanolic fresh and aged garlic extracts and their effects on cerebral ischemia," Food Chem, 140 (1-2). 343-352. Sep 1.2013.

[14] Boado, R.J.andPardridge, W.M., "Glucose deprivation and hypoxia increase the expression of the GLUT1 glucose transporter via a specific mRNA cis-acting regulatory element," J Neurochem, 80 (3). 552-554. Feb. 2002.

[15] Bruckner, B.A., Ammini, C.V., Otal, M.P., Raizada, M.K. and Stacpoole, P.W., "Regulation of brain glucose transporters by glucose and oxygen deprivation," Metabolism, 48 (4). 422-431. Apr.1999.

[16] Shao, Y., Wellman, T.L., Lounsbury, K.M. and Zhao, F.Q., "Differential regulation of GLUT1 and GLUT8 expression by hypoxia in mammary epithelial cells," Am J Physiol Regul Integr Comp Physiol, 307 (3). R237-247. Aug 1.2014.

[17] Urabe, T., Hattori, N., Nagamatsu, S., Sawa, H.andMizuno, Y., "Expression of glucose transporters in rat brain following transient focal ischemic injury," J Neurochem, 67 (1). 265-271. Jul.1996.

[18] Chavez, J.C. and LaManna, J.C., "Activation of hypoxia-inducible factor-1 in the rat cerebral cortex after transient global ischemia: potential role of insulin-like growth factor-1," J Neurosci, 22 (20). 8922-8931. Oct 15.2002.

[19] Lipton, P., "Ischemic cell death in brain neurons," Physiol Rev, 79 (4). 1431-1568. Oct.1999.

[20] Thoren, A.E., Helps, S.C., Nilsson, M.andSims, N.R., "The metabolism of C-glucose by neurons and astrocytes in brain subregions following focal cerebral ischemia in rats," $J$ Neurochem, 97 (4). 968-978. May. 2006.

[21] Iwabuchi, S. and Kawahara, K., "Inducible astrocytic glucose transporter-3 contributes to the enhanced storage of intracellular glycogen during reperfusion after ischemia," Neurochem Int, 59 (2). 319-325. Aug.2011.

[22] Bergeron, M., Yu, A.Y., Solway, K.E., Semenza, G.L. and Sharp, F.R., "Induction of hypoxia-inducible factor-1 (HIF-1) and its target genes following focal ischaemia in rat brain," Eur $J$ Neurosci, 11 (12). 4159-4170. Dec.1999.

[23] Zhang, B., Yin, C.P., Zhao, Q.andYue, S.W., "Upregulation of HIF-1alpha by Hypoxia Protect Neuroblastoma Cells from Apoptosis by Promoting Survivin Expression," Asian Pac J Cancer Prev, 15 (19). 8251-8257. 2014.

[24] Sher, A., Fakhar-ul-Mahmood, M., Shah, S.N., Bukhsh, S. and Murtaza, G., "Effect of garlic extract on blood glucose level and 
lipid profile in normal and alloxan diabetic rabbits," Adv Clin Exp Med, 21 (6). 705-711. Nov-Dec.2012.

[25] Gundimeda, U., McNeill, T.H., Elhiani, A.A., Schiffman, J.E., Hinton, D.R. and Gopalakrishna, R., "Green tea polyphenols precondition against cell death induced by oxygen-glucose deprivation via stimulation of laminin receptor, generation of reactive oxygen species, and activation of protein kinase Cepsilon," J Biol Chem, 287 (41). 34694-34708. Oct 5.2012.
[26] Guo, S., Bragina, O., Xu, Y., Cao, Z., Chen, H., Zhou, B., Morgan, M., Lin, Y., Jiang, B.H., Liu, K.J. and Shi, H., "Glucose upregulates HIF-1 alpha expression in primary cortical neurons in response to hypoxia through maintaining cellular redox status," $J$ Neurochem, 105 (5). 1849-1860. Jun. 2008.

[27] Matsutomo, T., Stark, T.D. and Hofmann, T., "In vitro activityguided identification of antioxidants in aged garlic extract," $J$ Agric Food Chem, 61 (12). 3059-3067. Mar 27.2012. 